\title{
Lie Symmetry Reductions and Exact Solutions to the Rosenau Equation
}

\author{
Ben Gao and Hongxia Tian \\ College of Mathematics, Taiyuan University of Technology, Taiyuan 030024, China \\ Correspondence should be addressed to Ben Gao; benzi0116@163.com
}

Received 4 October 2014; Revised 18 November 2014; Accepted 18 November 2014; Published 14 December 2014

Academic Editor: Boris G. Konopelchenko

Copyright (C) 2014 B. Gao and H. Tian. This is an open access article distributed under the Creative Commons Attribution License, which permits unrestricted use, distribution, and reproduction in any medium, provided the original work is properly cited.

The Lie symmetry analysis is performed on the Rosenau equation which arises in modeling many physical phenomena. The similarity reductions and exact solutions are presented. Then the exact analytic solutions are considered by the power series method.

\section{Introduction}

Nonlinear partial differential equations (PDEs) arising in many physical fields such as condensed matter physics, fluid mechanics, and plasma physics and optics exhibit a rich variety of nonlinear phenomena. It is known that to find exact solutions of the PDEs is always one of the central themes in mathematics and physics. A wealth of methods have been developed to find these exact physically significant solutions of a PDE though it is rather difficult. Some of the most important methods are the inverse scattering method [1], Hirota bilinear method [2], Darboux and Bäcklund transformations [3], Lie symmetry analysis [4-6], CK method [7, 8], and so forth. As is well known, the Lie group method is a powerful and direct approach to construct exact solutions of nonlinear differential equations. Furthermore, based on the Lie group method, many types of exact solutions of PDEs can be obtained, such as the traveling wave solutions, similarity solutions, soliton wave solutions, and fundamental solutions $[9,10]$.

The main idea of Lie group method is to transform solutions of a system of differential equations to other solutions. Once one has determined the symmetry group of a system of differential equations, a number of applications become available. Then one can directly use the defining property of such a group and construct new solutions to the system from known ones.
In the present paper, based on the Lie group method, we will consider an important equation, which is the Rosenau equation $[11,12]$ with the form

$$
u_{t}+u_{x}+u u_{x}+u_{x x x t}=0
$$

where $u=u(x, t)$ is the unknown real function.

Equation (1) appears in a wide variety of physical applications. It can investigate the dynamics of dense discrete systems in the case of wave-wave and wave-wall interactions that cannot be described using the well-known $\mathrm{KdV}$ equation. So it is important to lucubrate the exact explicit solutions and similarity reductions for this equation. A number of works have been devoted to study the Rosenau equation such as decay and scattering of small amplitude solution [13], posteriori error estimates [14], and conservative difference scheme [15]. However, as the authors knew, the Lie symmetry analysis of (1) is left as open problems.

The outline of this paper is as follows: in Section 2, we perform Lie symmetry analysis for the Rosenau equation; in Section 3, we discuss the Lie symmetry group of (1); in Section 4, we deal with the similarity reductions of (1) using Lie group method and provide exact solutions of the equation based on the Lie group method; in Section 5, the exact solutions for the reduced equation are obtained by using the power series method; and in Section 6, we summarize our results and make closing remarks. 


\section{Lie Symmetry Analysis of the Rosenau Equation}

In this section, we perform Lie symmetry analysis for (1) and obtain its infinitesimal generator and commutation table of Lie algebra.

First of all, let us consider a one-parameter Lie group of infinitesimal transformation:

$$
\begin{gathered}
x \longrightarrow x+\epsilon \quad \xi(x, t, u), \\
t \longrightarrow t+\epsilon \quad \tau(x, t, u), \\
u \longrightarrow u+\epsilon \quad \phi(x, t, u),
\end{gathered}
$$

with a small parameter $\epsilon \ll 1$. The vector field associated with the above group of transformations can be written as

$$
V=\xi(x, t, u) \frac{\partial}{\partial x}+\tau(x, t, u) \frac{\partial}{\partial t}+\phi(x, t, u) \frac{\partial}{\partial u} .
$$

The symmetry group of (1) will be generated by the vector field of the form (3). Applying the fourth prolongation $\mathrm{pr}^{(4)} V$ to $(1)$, we find that the coefficient functions $\xi(x, t, u), \tau(x, t, u)$, and $\phi(x, t, u)$ must satisfy the symmetry condition

$$
u_{x} \phi+(1+u) \phi^{x}+\phi^{t}+\phi^{x x x t}=0,
$$

where $\phi^{x}, \phi^{t}$, and $\phi^{x x x t}$ are the coefficients of $\mathrm{pr}^{(4)} V$. Furthermore, we have

$$
\begin{gathered}
\phi^{t}=D_{t} \phi-u_{x} D_{t} \xi-u_{t} D_{t} \tau, \\
\phi^{x}=D_{x} \phi-u_{x} D_{x} \xi-u_{t} D_{x} \tau, \\
\phi^{x x x t}=D_{t} D_{x}^{3}\left(\phi-\xi u_{x}-\tau u_{t}\right)+\xi u_{x x x x t}+\tau u_{x x x t},
\end{gathered}
$$

where $D_{x}, D_{t}$ are the total derivatives with respect to $x$ and $t$, respectively.

Substituting (5) into (4), equating the coefficients of the various monomials in the first, second, and the other order partial derivatives and various powers of $u$, we can find the following equations for the symmetry group of the Rosenau equation:

$$
\begin{gathered}
\xi_{x}=\xi_{t}=\xi_{u}=0, \\
\tau_{x}=\tau_{u}=0, \quad \tau_{t t}=0, \\
\phi=-\tau_{t}(1+u) .
\end{gathered}
$$

Solving (6), we obtain

$$
\begin{gathered}
\xi=c_{1}, \\
\tau=c_{2} t+c_{3}, \\
\phi=-c_{2}(1+u),
\end{gathered}
$$

where $c_{1}, c_{2}$, and $c_{3}$ are arbitrary constants.

Hence, the Lie algebra of infinitesimal symmetries of (1) is spanned by the following vector fields

$$
V_{1}=\frac{\partial}{\partial x}, \quad V_{2}=t \frac{\partial}{\partial t}-(1+u) \frac{\partial}{\partial u}, \quad V_{3}=\frac{\partial}{\partial t} .
$$

Then, all of the infinitesimal generators of (1) can be expressed as

$$
V=c_{1} V_{1}+c_{2} V_{2}+c_{3} V_{3} .
$$

It is easy to verify that $\left\{V_{1}, V_{2}, V_{3}\right\}$ is closed under the Lie bracket. In fact, we have

$$
\begin{gathered}
{\left[V_{1}, V_{1}\right]=\left[V_{2}, V_{2}\right]=\left[V_{3}, V_{3}\right]=0,} \\
{\left[V_{1}, V_{2}\right]=-\left[V_{2}, V_{1}\right]=\left[V_{1}, V_{3}\right]=-\left[V_{3}, V_{1}\right]=0,} \\
{\left[V_{2}, V_{3}\right]=-\left[V_{3}, V_{2}\right]=-V_{3} .}
\end{gathered}
$$

\section{Symmetry Groups of the Rosenau Equation}

In this section, in order to get exact solutions from a known solution of (1), we should find the one-parameter symmetry groups $G_{i}:(x, t, u) \rightarrow(\tilde{x}, \tilde{t}, \tilde{u})$ of corresponding infinitesimal generators. To get the Lie symmetry groups, we should solve the following initial problems of ordinary differential equations:

$$
\begin{aligned}
& \frac{d(\tilde{x}, \tilde{t}, \tilde{u})}{d \epsilon}=(\xi, \tau, \phi), \\
& \left.(\tilde{x}, \tilde{t}, \tilde{u})\right|_{\epsilon=0}=(x, t, u),
\end{aligned}
$$

where $\xi=\xi(\tilde{x}, \tilde{t}, \tilde{u}), \tau=\tau(\tilde{x}, \tilde{t}, \tilde{u}), \phi=\phi(\tilde{x}, \tilde{t}, \tilde{u})$, and $\epsilon$ is a group parameter.

For the infinitesimal generator $V=c_{1} V_{1}+c_{2} V_{2}+c_{3} V_{3}$, we will take the following different values to obtain the corresponding infinitesimal generators.

Case 1. Consider $c_{1}=1, c_{2}=c_{3}=0$, and the infinitesimal generator is $V_{1}=\partial / \partial x$.

Case 2. Consider $c_{2}=1, c_{1}=c_{3}=0$, and the infinitesimal generator is $V_{2}=t(\partial / \partial t)-(1+u)(\partial / \partial u)$.

Case 3. Consider $c_{3}=1, c_{1}=c_{2}=0$, and the infinitesimal generator is $V_{3}=\partial / \partial t$.

Case 4. Consider $c_{1}=c_{2}=1, c_{3}=0$, and the infinitesimal generator is $V_{1}+V_{2}=(\partial / \partial x)+t(\partial / \partial t)-(1+u)(\partial / \partial u)$.

Case 5. Consider $c_{2}=c_{3}=1, c_{1}=0$, and the infinitesimal generator is $V_{2}+V_{3}=(1+t)(\partial / \partial t)-(1+u)(\partial / \partial u)$.

Case 6. Consider $c_{2}=0, c_{1}=c \neq 0, c_{3}=1$, and the infinitesimal generator is $c V_{1}+V_{3}=c(\partial / \partial x)+(\partial / \partial t)$.

The one-parameter groups $G_{i}$ of the above corresponding infinitesimal generators are given as follows:

$$
\begin{aligned}
& G_{1}:(x, t, u) \rightarrow(x+\epsilon, t, u), \\
& G_{2}:(x, t, u) \rightarrow\left(x, e^{\epsilon} t,(1+u) e^{-\epsilon}-1\right), \\
& G_{3}:(x, t, u) \rightarrow(x, t+\epsilon, u), \\
& G_{4}:(x, t, u) \rightarrow\left(x+\epsilon, e^{\epsilon} t,(1+u) e^{-\epsilon}-1\right), \\
& G_{5}:(x, t, u) \rightarrow\left(x,(1+t) e^{\epsilon}-1,(1+u) e^{-\epsilon}-1\right), \\
& G_{6}:(x, t, u) \rightarrow(x+c \epsilon, t+\epsilon, u),
\end{aligned}
$$


where $\epsilon$ is any real number. We observe that $G_{1}$ is a space translation, $G_{3}$ is a time translation, $G_{6}$ is a space-time translation, and the groups $G_{i}(i=2,4,5)$ are genuinely local groups of transformation. Their appearance is far from obvious from basic physical principles, but they are very important for us to study the exact solutions of PDEs.

Since each $G_{i}(i=1,2,3,4,5,6)$ is a symmetry group, it implies that if $u=f(x, t)$ is a solution of (1), then $u^{(1)}, u^{(2)}$, $u^{(3)}, u^{(4)}, u^{(5)}$, and $u^{(6)}$ as follows are solutions of (1) as well:

$$
\begin{aligned}
& u^{(1)}=f(x-\epsilon, t), \\
& u^{(2)}=e^{-\epsilon} f\left(x, e^{-\epsilon} t\right)+e^{-\epsilon}-1, \\
& u^{(3)}=f(x, t-\epsilon), \\
& u^{(4)}=e^{-\epsilon} f\left(x-\epsilon, e^{-\epsilon} t\right)+e^{-\epsilon}-1, \\
& u^{(5)}=e^{-\epsilon} f\left(x,(1+t) e^{-\epsilon}-1\right)+e^{-\epsilon}-1, \\
& u^{(6)}=f(x-c \epsilon, t-\epsilon),
\end{aligned}
$$

where $\epsilon$ is any real number.

\section{Symmetry Reductions and Exact Solutions of the Rosenau Equation}

In the previous sections, we obtained the infinitesimal generators. In this section, we will get similarity variables and their reduction equations and obtain similarity solutions by solving the reduction equations.

Case 1. For the infinitesimal generator $V_{1}=\partial / \partial x$, we have the following similarity variables:

$$
\xi=t, \quad \omega=u,
$$

and the group-invariant solution is $\omega=f(\xi)$; that is,

$$
u=f(t) \text {. }
$$

Substituting (14) into (1), we obtain the following reduction equation:

$$
f^{\prime}=0
$$

where $f^{\prime}=d f / d \xi$. Therefore, (1) has a solution $u=c$, where $c$ is arbitrary constant. Obviously, the solution is not meaningful.

Case 2. For the infinitesimal generator $V_{2}=t(\partial / \partial t)-(1+$ $u)(\partial / \partial u)$, we have the following similarity variables:

$$
\xi=x, \quad \omega=(1+u) t,
$$

and the group-invariant solution is $\omega=f(\xi)$; that is,

$$
u=t^{-1} f(x)-1 \text {. }
$$

Substituting (17) into (1), we obtain the following reduction equation:

$$
-f+f f^{\prime}-f^{(3)}=0
$$

where $f^{\prime}=d f / d \xi$.
Case 3. For the infinitesimal generator $V_{3}=\partial / \partial t$, we have the following similarity variables:

$$
\xi=x, \quad \omega=u,
$$

and the group-invariant solution is $\omega=f(\xi)$; that is,

$$
u=f(x) \text {. }
$$

Substituting (20) into (1), we obtain the following reduction equation:

$$
f^{\prime}+f f^{\prime}=0
$$

where $f^{\prime}=d f / d \xi$. Therefore, (1) has a solution $u=c$, where $c$ is arbitrary constant.

Case 4. For the infinitesimal generator $V_{1}+V_{2}=(\partial / \partial x)+$ $t(\partial / \partial t)-(1+u)(\partial / \partial u)$, we have the following similarity variables:

$$
\xi=t e^{-x}, \quad \omega=(1+u) e^{x},
$$

and the group-invariant solution is $\omega=f(\xi)$; that is,

$$
u=e^{-x} f\left(t e^{-x}\right)-1 .
$$

Substituting (23) into (1), we obtain the following reduction equation:

$$
f^{2}+7 f^{\prime}+\xi f f^{\prime}+19 \xi f^{\prime \prime}+9 \xi^{2} f^{(3)}+\xi^{3} f^{(4)}=0
$$

where $f^{\prime}=d f / d \xi$.

Case 5. For the infinitesimal generator $V_{2}+V_{3}=(1+t)(\partial / \partial t)-$ $(1+u)(\partial / \partial u)$, we have the following similarity variables:

$$
\xi=x, \quad \omega=t+(1+t) u,
$$

and the group-invariant solution is $\omega=f(\xi)$; that is,

$$
u=\frac{f(x)-t}{1+t} .
$$

Substituting (26) into (1), we obtain the following reduction equation:

$$
-f+f^{\prime}+f f^{\prime}-f^{(3)}-1=0,
$$

where $f^{\prime}=d f / d \xi$.

Case 6. For the infinitesimal generator $c V_{1}+V_{3}=c(\partial / \partial x)+$ $(\partial / \partial t)$, we have the following similarity variables:

$$
\xi=x-c t, \quad \omega=u,
$$

and the group-invariant solution is $\omega=f(\xi)$; that is,

$$
u=f(x-c t) \text {. }
$$

Substituting (29) into (1), we obtain the following reduction equation:

$$
(1-c) f^{\prime}+f f^{\prime}-c f^{(4)}=0,
$$

where $f^{\prime}=d f / d \xi$.

Remark 1. Note that the reduced equations such as (18) and (24) are all higher-order nonlinear or nonautonomous ODEs; we will deal with such equations in the next section. 


\section{The Exact Power Series Solutions}

In this section, we want to detect the explicit solutions expressed in terms of elementary or known functions of mathematical physics, in terms of quadratures, and so on. But this is not always the case, even for simple semilinear PDEs. However, we know that the power series can be used to solve differential equations, including many complicated differential equations with nonconstant coefficients [16]. In this section, we will consider the exact analytic solutions to the reduced equations by using the power series method. Once we get the exact analytic solutions of the reduced equations (ODEs), the exact power series solutions to the original PDEs are obtained. In this section, we will consider the exact solutions of (18), (24), (27), and (30).

5.1. Exact Analytic Solutions to (18). Now, we seek a solution of (18) in a power series of the form

$$
f(\xi)=\sum_{n=0}^{\infty} c_{n} \xi^{n} .
$$

Substituting (31) into (18), we have

$$
\begin{aligned}
-c_{0} & -\sum_{n=1}^{\infty} c_{n} \xi^{n}+c_{0} c_{1} \\
& +\sum_{n=1}^{\infty}\left(\sum_{k=0}^{n}(n+1-k) c_{k} c_{n+1-k}\right) \xi^{n}-6 c_{3} \\
& -\sum_{n=1}^{\infty}(n+1)(n+2)(n+3) c_{n+3} \xi^{n}=0 .
\end{aligned}
$$

From (32), comparing coefficients, for $n=0$, we obtain

$$
c_{3}=\frac{1}{6} c_{0}\left(c_{1}-1\right) \text {. }
$$

Generally, for $n \geq 1$, we have

$$
\begin{aligned}
c_{n+3}= & \frac{1}{(n+1)(n+2)(n+3)} \\
& \times\left(-c_{n}+\sum_{k=0}^{n}(n+1-k) c_{k} c_{n+1-k}\right) .
\end{aligned}
$$

From (33) and (34), we can get all the coefficients $c_{n}(n \geq 3)$ of the power series (31); for example,

$$
\begin{aligned}
& c_{4}=\frac{1}{24}\left[c_{1}\left(c_{1}-1\right)+2 c_{0} c_{2}\right], \\
& c_{5}=\frac{1}{60}\left[3\left(c_{0} c_{3}+c_{1} c_{2}\right)-c_{2}\right] .
\end{aligned}
$$

Thus, for arbitrary chosen constants $c_{0}, c_{1}$, and $c_{2}$, the other terms of the sequence $\left\{c_{n}\right\}_{n=0}^{\infty}$ can be determined successively from (33) and (34) in a unique manner. This implies that, for (18), there exists a power series solution (31) with the coefficients given by (33) and (34). Furthermore, it is easy to prove the convergence of the power series (31) with the coefficients given by (33) and (34) (see, e.g., $[17,18]$ ). Therefore, this power series solution (31) to (18) is an exact analytic solution.

Hence, the power series solution of (18) can be written as follows:

$$
\begin{aligned}
f(\xi)= & c_{0}+c_{1} \xi+c_{2} \xi^{2}+c_{3} \xi^{3}+\sum_{n=1}^{\infty} c_{n+3} \xi^{n+3} \\
= & c_{0}+c_{1} \xi+c_{2} \xi^{2}+\frac{1}{6} c_{0}\left(c_{1}-1\right) \xi^{3} \\
& +\sum_{n=1}^{\infty} \frac{1}{(n+1)(n+2)(n+3)} \\
& \times\left(-c_{n}+\sum_{k=0}^{n}(n+1-k) c_{k} c_{n+1-k}\right) \xi^{n+3}
\end{aligned}
$$

Thus, the exact power series solution of (1) is

$$
\begin{aligned}
u(x, t)= & t^{-1}\left(c_{0}+c_{1} x+c_{2} x^{2}+c_{3} x^{3}+\sum_{n=1}^{\infty} c_{n+3} x^{n+3}\right)-1 \\
= & c_{0} t^{-1}+c_{1} t^{-1} x+c_{2} t^{-1} x^{2}+\frac{1}{6} c_{0}\left(c_{1}-1\right) t^{-1} x^{3} \\
& +\sum_{n=1}^{\infty} \frac{1}{(n+1)(n+2)(n+3)} \\
& \times\left(-c_{n}+\sum_{k=0}^{n}(n+1-k) c_{k} c_{n+1-k}\right) \\
& \times t^{-1} x^{n+3}-1
\end{aligned}
$$

where $c_{0}, c_{1}$, and $c_{2}$ are arbitrary constants; the other coefficients $c_{n}(n \geq 3)$ can be determined successively from (33) and (34).

In physical applications, it will be convenient to write the solution of (1) in the approximate form

$$
\begin{aligned}
u(x, t)= & -1+c_{0} t^{-1}+c_{1} t^{-1} x+c_{2} t^{-1} x^{2} \\
& +\frac{1}{6} c_{0}\left(c_{1}-1\right) t^{-1} x^{3}+\frac{1}{24}\left[c_{1}\left(c_{1}-1\right)+2 c_{0} c_{2}\right] t^{-1} x^{4} \\
& +\frac{1}{60}\left[3\left(c_{0} c_{3}+c_{1} c_{2}\right)-c_{2}\right] t^{-1} x^{5}+\cdots,
\end{aligned}
$$

in terms of the above computation. 
5.2. Exact Analytic Solutions to (24). Similarly, we seek a solution of (24) in a power series of the form (31). Substituting it into (24) and comparing coefficients, we obtain

$$
\begin{aligned}
& c_{1}=-\frac{1}{7} c_{0}^{2}, \\
& c_{2}=-\frac{3}{52} c_{0} c_{1}, \\
& c_{3}=-\frac{2}{189}\left(2 c_{0} c_{2}+c_{1}^{2}\right) .
\end{aligned}
$$

Generally, for $n \geq 0$, we have

$$
c_{n+4}=-\frac{\sum_{k=0}^{n+2}(n+4-k) c_{k} c_{n+3-k}+c_{0} c_{n+3}}{(n+4)\left(7+(n+3)\left(n^{2}+12 n+39\right)\right)} .
$$

In view of (39)-(42), we can get all the coefficients $c_{n}(n \geq 1)$ of the power series (31); for example,

$$
c_{4}=-\frac{5}{496}\left(c_{1} c_{2}+c_{0} c_{3}\right)
$$

Thus, for arbitrary chosen constant $c_{0}$, the other terms of the sequence $\left\{c_{n}\right\}_{n=0}^{\infty}$ can be determined successively from (39)(42) in a unique manner. This implies that, for (24), there exists a power series solution (31) with the coefficients given by (39)-(42).

Therefore, the power series solution of (24) can be written as follows:

$$
\begin{aligned}
f(\xi)= & c_{0}-\frac{1}{7} c_{0}^{2} \xi-\frac{3}{52} c_{0} c_{1} \xi^{2}-\frac{2}{189}\left(2 c_{0} c_{2}+c_{1}^{2}\right) \xi^{3} \\
& -\sum_{n=0}^{\infty} \frac{\sum_{k=0}^{n+2}(n+4-k) c_{k} c_{n+3-k}+c_{0} c_{n+3}}{(n+4)\left(7+(n+3)\left(n^{2}+12 n+39\right)\right)} \xi^{n+4} .
\end{aligned}
$$

Accordingly we have the exact solution to (1) being

$$
\begin{aligned}
u(x, t)= & c_{0} e^{-x}-\frac{1}{7} c_{0}^{2} t e^{-2 x}-\frac{3}{52} c_{0} c_{1} t^{2} e^{-3 x} \\
& -\frac{2}{189}\left(2 c_{0} c_{2}+c_{1}^{2}\right) t^{3} e^{-4 x} \\
& -\sum_{n=0}^{\infty} \frac{\sum_{k=0}^{n+2}(n+4-k) c_{k} c_{n+3-k}+c_{0} c_{n+3}}{(n+4)\left(7+(n+3)\left(n^{2}+12 n+39\right)\right)} \\
& \quad \times t^{n+4} e^{-(n+5) x}-1,
\end{aligned}
$$

where $c_{0}$ is arbitrary constant; the other terms $c_{n}(1,2,3, \ldots)$ are given by (39)-(42) successively.

5.3. Exact Analytic Solutions to (27). Now, we seek a solution of (27) in a power series of the form (31). Substituting (31) into (27) and comparing coefficients, we obtain

$$
c_{3}=\frac{1}{6}\left(c_{0}+1\right)\left(c_{1}-1\right) .
$$

For $n \geq 1$, we have

$$
\begin{aligned}
c_{n+3}= & \frac{1}{(n+1)(n+2)(n+3)} \\
& \times\left(-c_{n}+(n+1) c_{n+1}+\sum_{k=0}^{n}(n+1-k) c_{k} c_{n+1-k}\right) .
\end{aligned}
$$

From (46) and (47), we can get all the coefficients $c_{n}(n \geq 3)$ of the power series (31) such as

$$
\begin{aligned}
& c_{4}=\frac{1}{24}\left[c_{1}\left(c_{1}-1\right)+2 c_{2}\left(c_{0}+1\right)\right], \\
& c_{5}=\frac{1}{60}\left[c_{2}\left(3 c_{1}-1\right)+3 c_{3}\left(c_{0}+1\right)\right] .
\end{aligned}
$$

Thus, for arbitrary chosen constants $c_{0}, c_{1}$, and $c_{2}$, the other terms of the sequence $\left\{c_{n}\right\}_{n=0}^{\infty}$ can be determined successively from (46) and (47) in a unique manner. This implies that, for (27), there exists a power series solution (31) with the coefficients given by (46) and (47).

The exact solution of (1) and the solution in the approximate form can be written in terms of the above computation. The details are omitted here.

5.4. Exact Analytic Solutions to (30). Similarly, we seek a solution of (30) in a power series of the form (31). Substituting it into (30) and comparing coefficients, we obtain

$$
\begin{array}{r}
c_{n+4}=\frac{\sum_{k=0}^{n}(n+1-k) c_{k} c_{n+1-k}+(1-c)(n+1) c_{n+1}}{c(n+1)(n+2)(n+3)(n+4)}, \\
n=0,1,2, \ldots
\end{array}
$$

In view of (49), we can get all the coefficients $c_{n}(n \geq 4)$ of the power series (31) such as

$$
\begin{aligned}
& c_{4}=\frac{c_{1}\left(c_{0}-c+1\right)}{24 c}, \\
& c_{5}=\frac{c_{1}^{2}+2 c_{2}\left(c_{0}-c+1\right)}{120 c}, \\
& c_{6}=\frac{c_{1} c_{2}+c_{3}\left(c_{0}-c+1\right)}{120 c}, \\
& c_{7}=\frac{2 c_{1} c_{3}+c_{2}^{2}+2 c_{4}\left(c_{0}-c+1\right)}{420 c} .
\end{aligned}
$$

Thus, for arbitrary chosen constants $c_{0}, c_{1}, c_{2}$, and $c_{3}$, the other terms of the sequence $\left\{c_{n}\right\}_{n=0}^{\infty}$ can be determined successively from (49) in a unique manner. Therefore, the power series solution of (30) can be written as follows:

$$
\begin{aligned}
& f(\xi)= c_{0}+c_{1} \xi+c_{2} \xi^{2}+c_{3} \xi^{3} \\
&+\sum_{n=0}^{\infty} \frac{\sum_{k=0}^{n}(n+1-k) c_{k} c_{n+1-k}+(1-c)(n+1) c_{n+1}}{c(n+1)(n+2)(n+3)(n+4)} \\
& \quad \times \xi^{n+4} .
\end{aligned}
$$


Accordingly, we have the exact traveling wave solution to (1) as follows:

$$
\begin{aligned}
& u(x, t)= c_{0}+c_{1}(x-c t)+c_{2}(x-c t)^{2}+c_{3}(x-c t)^{3} \\
&+\sum_{n=0}^{\infty} \frac{\sum_{k=0}^{n}(n+1-k) c_{k} c_{n+1-k}+(1-c)(n+1) c_{n+1}}{c(n+1)(n+2)(n+3)(n+4)} \\
& \quad \times(x-c t)^{n+4},
\end{aligned}
$$

where $c_{i}(i=0,1,2,3)$ are arbitrary constants; the other terms $c_{n+4}(n=0,1,2, \ldots)$ are given by (49) successively.

Remark 2. By using the integration of ordinary differential equations (ODEs), we know that if we get a one-parameter symmetry group of an ODE, then we can reduce the order of the equation by one. But we note that such reduced ODEs are more complicated than the original equation in addition to some special cases. In view of this, we can see that the power series method is a useful tool of solving such higher-order nonlinear or nonautonomous ODEs.

\section{Conclusions}

In this paper, we study the symmetry reductions and exact solutions of the Rosenau equation by using the classical Lie group method. First, we perform Lie symmetry analysis for the Rosenau equation and get its infinitesimal generator and commutation table of Lie algebra. Then, we discuss the Lie symmetry groups of the Rosenau equation. Moreover, using similarity variables to obtain reduction equations and solving the reduction equation, we obtain all the group-invariant solutions to the equation. Then the exact analytic solutions are investigated by using the power series method. Especially, the similarity reductions and exact solutions are given for the first time in this paper. The physical significance of the solutions is considered from the transformation group point of view. These similarity solutions possess significant features in the nonlinear mechanics aspects of the work.

\section{Conflict of Interests}

The authors declare that there is no conflict of interests regarding the publication of this paper.

\section{Acknowledgment}

This paper is supported by the Natural Science Foundation of Shanxi (no. 2014021010-1).

\section{References}

[1] C. S. Gardner, J. M. Greene, M. D. Kruskal, and R. M. Miura, "Method for solving the Korteweg-deVries equation," Physical Review Letters, vol. 19, no. 19, pp. 1095-1097, 1967.

[2] R. Hirota and J. Satsuma, "A variety of nonlinear network equations generated from the Bäcklund transformation for the Toda lattice," Progress of Theoretical Physics, no. 59, pp. 64-100, 1976.
[3] Y. S. Li, Soliton and Integrable Systems, Advanced Series in Nonlinear Science, Shanghai Scientific and Technological Education Publishing House, Shanghai, China, 1999 (Chinese).

[4] P. J. Olver, Applications of Lie Groups to Differential Equations, vol. 107 of Graduate Texts in Mathematics, Springer, New York, NY, USA, 1993.

[5] G. W. Bluman and S. Kumei, Symmetries and Differential Equations, Springer, World Publishing Corporation, 1989.

[6] B. J. Cantwell, Introduction to Symmetry Analysis, Cambridge Texts in Applied Mathematics, Cambridge University Press, Cambridge, UK, 2002.

[7] P. A. Clarkson and M. D. Kruskal, "New similarity reductions of the Boussinesq equation," Journal of Mathematical Physics, vol. 30, no. 10, pp. 2201-2213, 1989.

[8] P. A. Clarkson, "New similarity solutions for the modified Boussinesq equation," Journal of Physics A: Mathematical and General, vol. 22, no. 13, pp. 2355-2367, 1989.

[9] M. Craddock and E. Platen, "Symmetry group methods for fundamental solutions," Journal of Differential Equations, vol. 207, no. 2, pp. 285-302, 2004.

[10] M. Craddock and K. A. Lennox, "Lie group symmetries as integral transforms of fundamental solutions," Journal of Differential Equations, vol. 232, no. 2, pp. 652-674, 2007.

[11] P. Rosenau, "A quasi-continuous description of a nonlinear transmission line," Physica Scripta, vol. 34, pp. 827-829, 1986.

[12] P. Rosenau, "Dynamics of dense discrete systems," Progress of Theoretical Physics, vol. 79, no. 5, pp. 1028-1042, 1988.

[13] H. Wang and S. Wang, "Decay and scattering of small solutions for Rosenau equations," Applied Mathematics and Computation, vol. 218, no. 1, pp. 115-123, 2011.

[14] S. M. Choo, S. K. Chung, and K. I. Kim, "A discontinuous Galerkin method for the Rosenau equation," Applied Numerical Mathematics, vol. 58, no. 6, pp. 783-799, 2008.

[15] K. Omrani, F. Abidi, T. Achouri, and N. Khiari, "A new conservative finite difference scheme for the Rosenau equation," Applied Mathematics and Computation, vol. 201, no. 1-2, pp. 35-43, 2008.

[16] N. H. Asmar, Partial Differential Equations with Fourier Series and Boundary Value Problems, China Machine Press, Beijing, China, 2nd edition, 2005.

[17] H. Liu and J. Li, "Lie symmetry analysis and exact solutions for the short pulse equation," Nonlinear Analysis: Theory, Methods \& Applications, vol. 71, no. 5-6, pp. 2126-2133, 2009.

[18] H. Liu, J. Li, and Q. Zhang, "Lie symmetry analysis and exact explicit solutions for general Burgers' equation," Journal of Computational and Applied Mathematics, vol. 228, no. 1, pp. 1-9, 2009. 


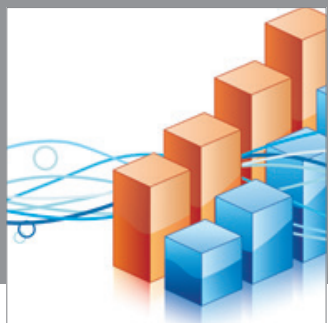

Advances in

Operations Research

mansans

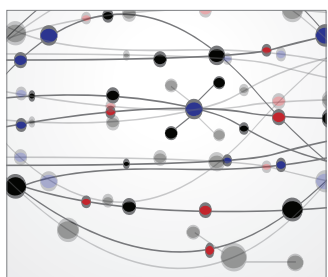

The Scientific World Journal
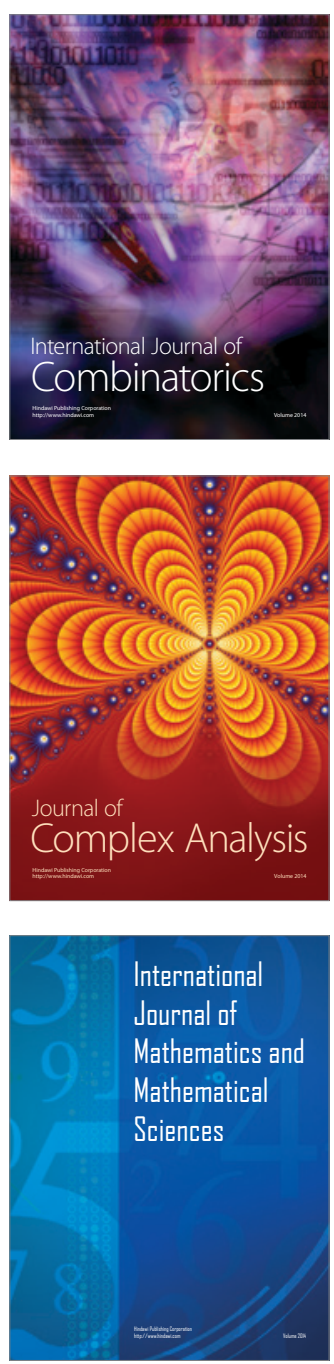
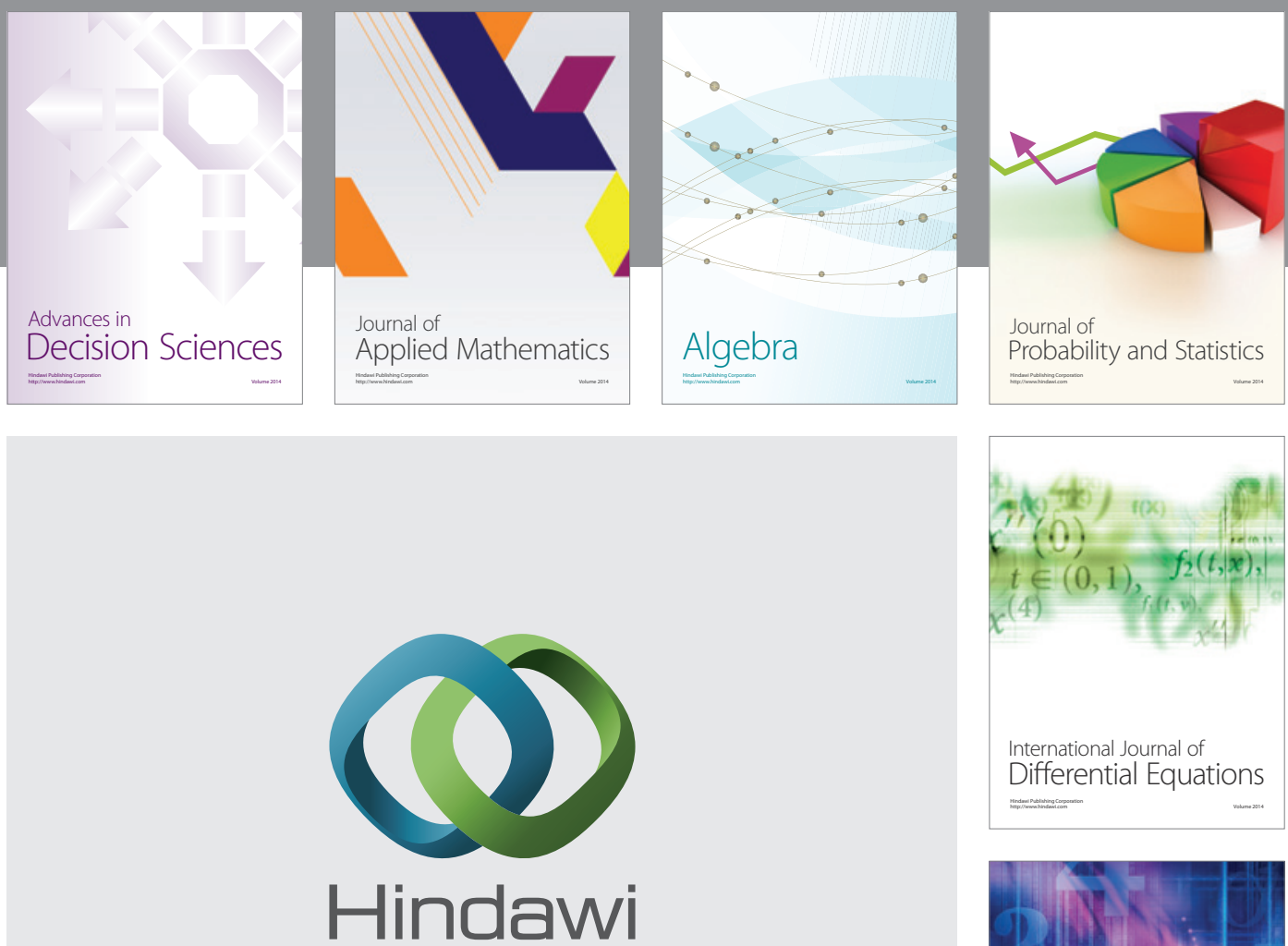

Submit your manuscripts at http://www.hindawi.com
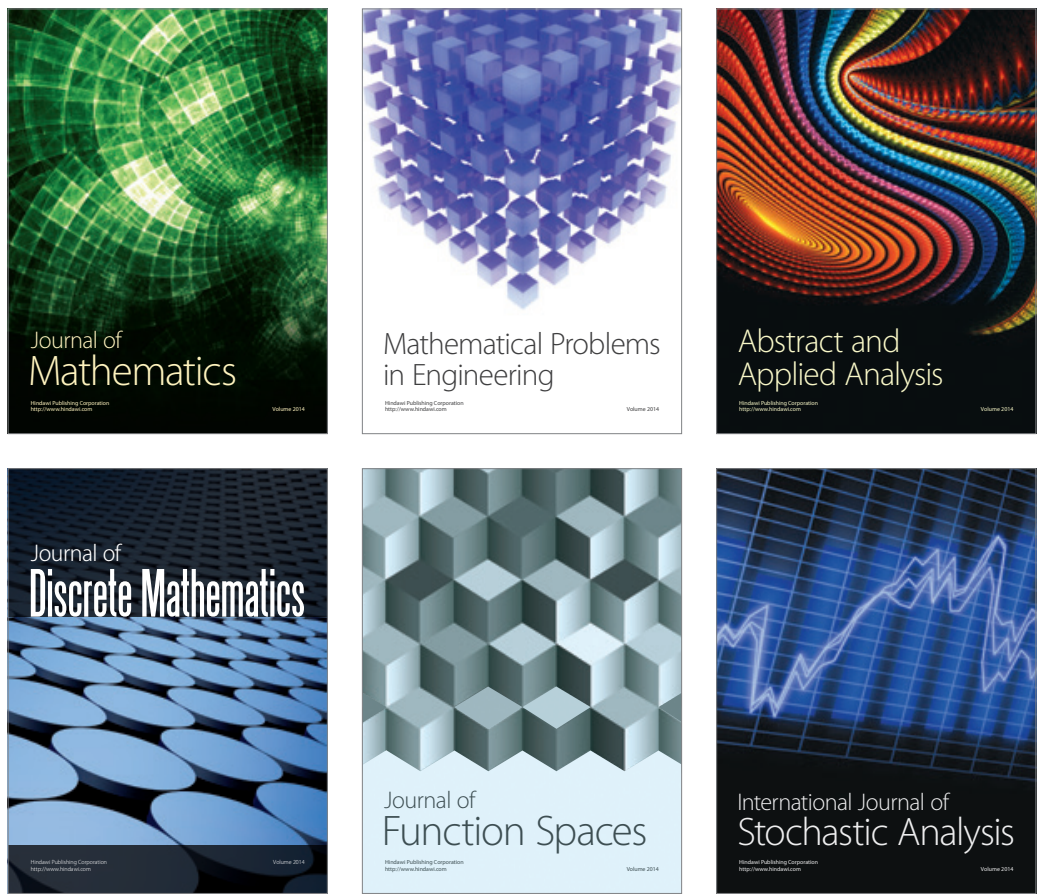

Journal of

Function Spaces

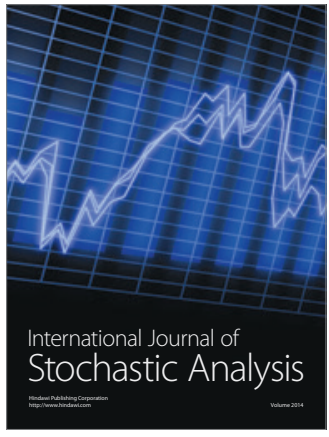

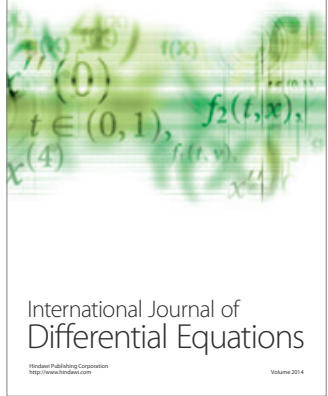
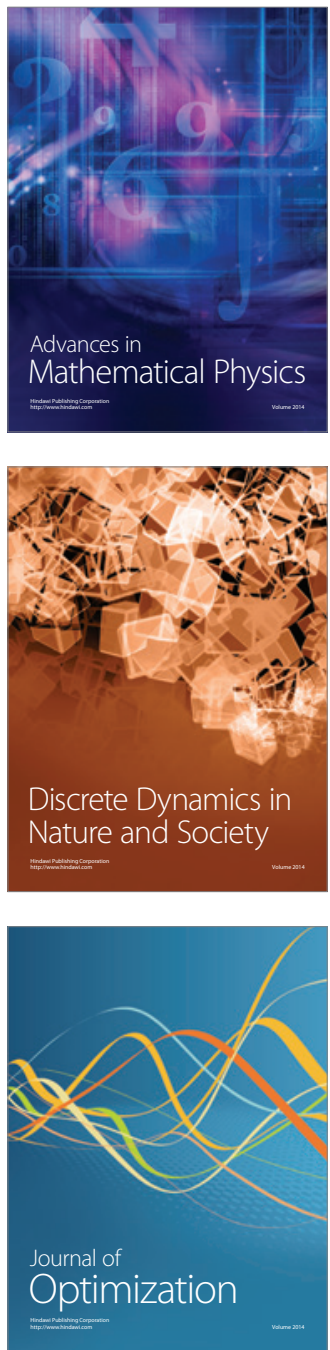